\title{
Estimation in an Exponentiated Half Logistic Distribution under Progressively Type-II Censoring
}

\author{
Suk-Bok Kang ${ }^{1, a}$, Jung-In Seo ${ }^{a}$ \\ ${ }^{a}$ Department of Statistics, Yeungnam University
}

\begin{abstract}
In this paper, we derive the maximum likelihood estimator(MLE) and some approximate maximum likelihood estimators(AMLEs) of the scale parameter in an exponentiated half logistic distribution based on progressively Type-II censored samples. We compare the proposed estimators in the sense of the mean squared error(MSE) through a Monte Carlo simulation for various censoring schemes. We also obtain the AMLEs of the reliability function.
\end{abstract}

Keywords: Approximate maximum likelihood estimator, exponentiated half logistic distribution, progressively Type-II censored sample, reliability.

\section{Introduction}

The probability density function(pdf) of the random variable $X$ having the exponentiated half logistic distribution is given by

$$
f(x)=\frac{\lambda}{\sigma}\left[\frac{1-\exp \left(-\frac{x}{\sigma}\right)}{1+\exp \left(-\frac{x}{\sigma}\right)}\right]^{\lambda-1} \frac{2 \exp \left(-\frac{x}{\sigma}\right)}{\left[1+\exp \left(-\frac{x}{\sigma}\right)\right]^{2}}, \quad x>0, \sigma, \lambda>0,
$$

where $\sigma$ is the scale parameter and $\lambda$ is known constant.

In special case, when $\lambda=1$, this distribution is the half logistic distribution. The half logistic distribution has been used quite extensively in reliability and survival analysis, particularly when the data is censored. Inferences for the half logistic distribution were discussed by several authors. Balakrishnan and Puthenpura (1986) obtained the best linear unbiased estimators of the location and scale parameters of the half logistic distribution through a linear functions of order statistics. Balakrishnan and Wong (1991) obtained the AMLEs for the location and scale parameters of the half logistic distribution with a Type-II right censored sample. Kang et al. (2008) derived the AMLEs and MLE of the scale parameter in a half logistic distribution based on progressively Type-II censored samples. Kang et al. (2009) derived the AMLEs and MLE of the scale parameter for the half logistic distribution under the proposed double hybrid censored samples. Recently, Arora et al. (2010) obtained the MLE and its asymptotic variance of the generalized half logistic distribution under Type-I progressive censoring with changing failure rates. They also obtained some results that include total expected waiting time in case of interval censoring schemes.

It has been noted that in most cases, the maximum likelihood method does not provide explicit estimators based on complete and censored samples. Especially, the maximum likelihood method does

\footnotetext{
${ }^{1}$ Corresponding author: Professor, Department of Statistics, Yeungnam University, Gyeongsan 712-749, Korea.

E-mail: sbkang@yu.ac.kr
} 
not admit explicit solutions when the sample is multiply or progressively censored. Hence it is desirable to develop some approximations to this maximum likelihood method that provide us with some estimators that are explicit functions of order statistics. The approximate maximum likelihood estimation method was first developed by Balakrishnan (1989) for the purpose of providing the explicit estimators of the scale parameter in the Rayleigh distribution. Balakrishnan et al. (2003) suggested the point and interval estimation for Gaussian distribution based on progressively Type-II censored samples. Balakrishnan et al. (2004) studied the point and interval estimation for the extreme value distribution based on progressively Type-II censored sample. Lin et al. (2006) discussed the MLEs of the parameters of the log-gamma distribution based on progressively Type-II censored samples, and they derived the AMLEs of the parameters and used them as initial values in the determination of the MLEs through the Newton-Raphson method. Seo and Kang (2007) derived the AMLEs for Rayleigh distribution based on progressively Type-II censored data. Han and Kang (2008) derived the AMLEs of the scale parameter and the location parameter in a double Rayleigh distribution based on multiply Type-II censored samples. Lee et al. (2008) derived several estimators of the scale parameter in a triangular distribution based on multiply Type-II censored samples by the approximate maximum likelihood estimation method. They also obtained the AMLEs of the reliability function through the use of the proposed estimators.

In this paper, we derive the MLE and some AMLEs of the scale parameter $\sigma$ under progressively Type-II censoring. The scale parameter is estimated by the approximate maximum likelihood estimation method using two different types of Taylor series expansions. We also obtain the MLE and the AMLEs of the reliability function using the proposed estimators. We compare the proposed estimators in the sense of the MSE through a Monte Carlo simulation for various censored samples.

\section{Maximum Likelihood Estimation}

In this section, we consider the MLE of the scale parameter in an exponentiated half logistic distribution under progressively Type-II censored data. Let us consider the following progressively Type-II censoring scheme. Suppose $n$ randomly selected units with exponentiated half logistic distribution in (1.1) were placed on a life test, only $m$ are completely observed until failure. At the time of the first failure, $r_{1}$ of the $n-1$ surviving units are randomly withdrawn (or censored) from the life testing experiment. At the time of the next failures, $r_{2}$ of the $n-2-r_{1}$ surviving units are randomly censored, and so on. Finally, at the time of the $m$ th failures, all the remaining $r_{m}=n-m-r_{1}-\cdots-r_{m-1}$ surviving units are censored.

Let

$$
X_{1: m: n} \leq X_{2: m: n} \leq \cdots \leq X_{m: m: n}
$$

denote such a progressively Type-II censored sample with $\left(r_{1}, \ldots, r_{m}\right)$ being the progressively censoring scheme. Note that the case $m=n$, in which case $r_{1}=\cdots=r_{m}=0$, corresponds to the complete sample situation, while the case $r_{1}=\cdots=r_{m-1}=0, r_{m}=n-m$ corresponds to the usual Type-II censored sample.

The likelihood function based on the progressively Type-II censored sample (2.1) is given by

$$
L=C \prod_{i=1}^{m} f\left(x_{i: m: n} ; \sigma, \lambda\right)\left[1-F\left(x_{i: m: n} ; \sigma, \lambda\right)\right]^{r_{i}},
$$

where $C=n\left(n-1-r_{1}\right)\left(n-2-r_{1}-r_{2}\right) \cdots\left(n-m+1-r_{1}-\cdots-r_{m-1}\right)$. 
Let $Z_{i: m: n}=X_{i: m: n} / \sigma$. From the Equation (2.2), the log-likelihood function may be rewritten as

$$
\log L=K-m \log \sigma+\sum_{i=1}^{m} \log f\left(z_{i: m: n}\right)+\sum_{i=1}^{m} r_{i} \log \left[1-F\left(z_{i: m: n}\right)\right],
$$

where $K$ is a constant.

Since the random variable $Z_{i: m: n}=X_{i: m: n} / \sigma$ has a standard exponentiated half logistic distribution with pdf and cdf

$$
\begin{aligned}
& f\left(z_{i: m: n}\right)=\lambda\left[\frac{1-\exp \left(-z_{i: m: n}\right)}{1+\exp \left(-z_{i: m: n}\right)}\right]^{\lambda-1} \frac{2 \exp \left(-z_{i: m: n}\right)}{\left[1+\exp \left(-z_{i: m: n}\right)\right]^{2}}, \\
& F\left(z_{i: m: n}\right)=\left[\frac{1-\exp \left(-z_{i: m: n}\right)}{1+\exp \left(-z_{i: m: n}\right)}\right]^{\lambda}, \quad z>0, \lambda>0,
\end{aligned}
$$

$f\left(z_{i: m: n}\right), f^{\prime}\left(z_{i: m: n}\right)$, and $F\left(z_{i: m: n}\right)$ are satisfied as

$$
\frac{f\left(z_{i: m: n}\right)}{F\left(z_{i: m: n}\right)}=\lambda \frac{2 \exp \left(-z_{i: m: n}\right)}{1-\exp \left(-2 z_{i: m: n}\right)}, \quad \frac{f^{\prime}\left(z_{i: m: n}\right)}{f\left(z_{i: m: n}\right)}=\left[\left(1-\frac{1}{\lambda}\right) \frac{f\left(z_{i: m: n}\right)}{F\left(z_{i: m: n}\right)}-\left[F\left(z_{i: m: n}\right)\right]^{\frac{1}{\lambda}}\right],
$$

From the Equation (2.3), we obtain the likelihood equation for $\sigma$ as follows;

$$
\begin{aligned}
\frac{\partial \log L}{\partial \sigma} & =-\frac{1}{\sigma}\left[m+\left(1-\frac{1}{\lambda}\right) \sum_{i=i}^{m} \frac{f\left(z_{i: m: n}\right)}{F\left(z_{i: m: n}\right)} z_{i: m: n}-\sum_{i=1}^{m}\left[F\left(z_{i: m: n}\right)\right]^{\frac{1}{\lambda}} z_{i: m: n}-\sum_{i=1}^{m} r_{i} \frac{f\left(z_{i: m: n}\right)}{1-F\left(z_{i: m: n}\right)} z_{i: m: n}\right] \\
& =0 .
\end{aligned}
$$

We can find the MLE $\hat{\sigma}$ of $\sigma$ that maximize the log-likelihood function in (2.3) by solving the Equation (2.4). Since the Equation (2.4) cannot be solved explicitly, some numerical methods must be employed. It is not easily to obtain the solution of the log-likelihood Equation (2.4) by NewtonRapshon method in this case. So we use the Bisection method to obtain the numerical solution of the Equation (2.4). Using this method, we evaluate the MSEs of the MLE of the scale parameter $\sigma$ for the sample size $n=10(10) 40$ and various censoring schemes based on progressively Type-II censored samples. These values are given in Table 5.

\section{Approximate Maximum Likelihood Estimation}

Since the likelihood equation (2.4) is very complicated, the equation does not admit an explicit solution for $\sigma$. Therefore, we will derive the AMLEs of $\sigma$ by approximating the likelihood equation (2.4).

Let

$$
\xi_{i}=F^{-1}\left(p_{i: m: n}\right)=\log \left[\frac{1+\left(p_{i: m: n}\right)^{\frac{1}{\lambda}}}{1-\left(p_{i: m: n}\right)^{\frac{1}{\lambda}}}\right]
$$

where $q_{i: m: n}=1-p_{i: m: n}$ and

$$
p_{i: m: n}=1-\prod_{j=m-i+1}^{m} \frac{j+r_{m-i+1}+\cdots+r_{m}}{j+1+r_{m-i+1}+\cdots+r_{m}}, \quad i=1, \ldots, m .
$$


First, we can approximate the following functions by

$$
\begin{aligned}
\frac{f\left(z_{i: m: n}\right)}{F\left(z_{i: m: n}\right)} z_{i: m: n} & \approx \alpha_{1 i}+\beta_{1 i} z_{i: m: n}, \\
{\left[F\left(z_{i: m: n}\right)\right]^{\frac{1}{\lambda}} z_{i: m: n} } & \approx \gamma_{1 i}+\eta_{1 i} z_{i: m: n}, \\
\frac{f\left(z_{i: m: n}\right)}{1-F\left(z_{i: m: n}\right)} z_{i: m: n} & \approx \kappa_{1 i}+\delta_{1 i} z_{i: m: n},
\end{aligned}
$$

where

$$
\begin{array}{ll}
\alpha_{1 i}=\frac{\left(\xi_{i: m: n}\right)^{2}}{p_{i: m: n}}\left[\frac{1+\left(p_{i: m: n}\right)^{\frac{2}{\lambda}}}{2\left(p_{i: m: n}\right)^{\frac{1}{\lambda}}}\right] f\left(\xi_{i: m: n}\right), & \beta_{1 i}=-\frac{f\left(\xi_{i: m: n}\right)}{p_{i: m: n}}\left[\frac{1+\left(p_{i: m: n}\right)^{\frac{2}{\lambda}}}{2\left(p_{i: m: n}\right)^{\frac{1}{\lambda}}} \xi_{i: m: n}-1\right], \\
\gamma_{1 i}=-\frac{\left(\xi_{i: m: n}\right)^{2}}{\lambda p_{i: m: n}}\left(p_{i: m: n}\right)^{\frac{1}{\lambda}} f\left(\xi_{i: m: n}\right), & \eta_{1 i}=\left(p_{i: m: n}\right)^{\frac{1}{\lambda}}\left[1+\frac{f\left(\xi_{i: m: n}\right) \xi_{i: m: n}}{\lambda p_{i: m: n}}\right], \\
\kappa_{1 i}=-\frac{\left(\xi_{i: m: n}\right)^{2}}{q_{i: m: n}}\left[f^{\prime}\left(\xi_{i: m: n}\right)+\frac{f^{2}\left(\xi_{i: m: n}\right)}{q_{i: m: n}}\right], & \delta_{1 i}=\frac{1}{q_{i: m: n}}\left[f\left(\xi_{i: m: n}\right)+\left(f^{\prime}\left(\xi_{i: m: n}\right)+\frac{f^{2}\left(\xi_{i: m: n}\right)}{q_{i: m: n}}\right) \xi_{i: m: n}\right] .
\end{array}
$$

By using the Equation (3.1), (3.2), and (3.3), we can approximate the likelihood equation (2.4) by

$$
\begin{aligned}
\frac{\partial \log L}{\partial \sigma} & \simeq-\frac{1}{\sigma}\left[m+\left(1-\frac{1}{\lambda}\right) \sum_{i=i}^{m}\left(\alpha_{1 i}+\beta_{1 i} z_{i: m: n}\right)-\sum_{i=1}^{m}\left(\gamma_{1 i}+\eta_{1 i} z_{i: m: n}\right)-\sum_{i=1}^{m} r_{i}\left(\kappa_{1 i}+\delta_{1 i} z_{i: m: n}\right)\right] \\
& =0
\end{aligned}
$$

Upon solving the Equation (3.4) for $\sigma$, we can derive an AMLE of $\sigma$ as follows;

$$
\tilde{\sigma}_{1}=\frac{\sum_{i=1}^{m} X_{i: m: n}\left[(1-1 / \lambda) \beta_{1 i}-\eta_{1 i}-r_{i} \delta_{1 i}\right]}{-m-\sum_{i=1}^{m}\left[(1-1 / \lambda) \alpha_{1 i}-\gamma_{1 i}-r_{i} K_{1 i}\right]} .
$$

For $\lambda>1$, since $\beta_{1 i}<0, \eta_{1 i}>0, \delta_{1 i}>0, \alpha_{1 i}>0, \gamma_{1 i}<0$, and $\kappa_{1 i}<0$, the estimator $\tilde{\sigma}_{1}$ is always positive.

Second, we can also approximate the other following functions by

$$
\begin{aligned}
\frac{f\left(z_{i: m: n}\right)}{F\left(z_{i: m: n}\right)} & \approx \alpha_{2 i}+\beta_{2 i} z_{i: m: n}, \\
{\left[F\left(z_{i: m: n}\right)\right]^{\frac{1}{\lambda}} } & \approx \gamma_{2 i}+\eta_{2 i} z_{i: m: n}, \\
\frac{f\left(z_{i: m: n}\right)}{1-F\left(z_{i: m: n}\right)} & \approx \kappa_{2 i}+\delta_{2 i} z_{i: m: n},
\end{aligned}
$$

where

$$
\begin{array}{ll}
\alpha_{2 i}=\frac{f\left(\xi_{i: m: n}\right)}{p_{i: m: n}}\left[\frac{1+\left(p_{i: m: n}\right)^{\frac{2}{\lambda}}}{2\left(p_{i: m: n}\right)^{\frac{1}{\lambda}}} \xi_{i: m: n}+1\right], & \beta_{2 i}=-\frac{f\left(\xi_{i: m: n}\right)}{p_{i: m: n}}\left[\frac{1+\left(p_{i: m: n}\right)^{\frac{2}{\lambda}}}{2\left(p_{i: m: n}\right)^{\frac{1}{\lambda}}}\right], \\
\gamma_{2 i}=\left(p_{i: m: n}\right)^{\frac{1}{\lambda}}\left[1-\frac{f\left(\xi_{i: m: n}\right) \xi_{i: m: n}}{\lambda p_{i: m: n}}\right], & \eta_{2 i}=\left(p_{i: m: n}\right)^{\frac{1}{\lambda}} \frac{f\left(\xi_{i: m: n}\right)}{\lambda p_{i: m: n}}, \\
\kappa_{2 i}=\frac{1}{q_{i: m: n}}\left[f\left(\xi_{i: m: n}\right)-\left(f^{\prime}\left(\xi_{i: m: n}\right)+\frac{f^{2}\left(\xi_{i: m: n}\right)}{q_{i: m: n}}\right) \xi_{i: m: n}\right], & \delta_{2 i}=\frac{1}{q_{i: m: n}}\left[f^{\prime}\left(\xi_{i: m: n}\right)+\frac{f^{2}\left(\xi_{i: m: n}\right)}{q_{i: m: n}}\right] .
\end{array}
$$


By using the Equation (3.6), (3.7), and (3.8), we can also approximate the likelihood equation in (2.4) by

$$
\begin{aligned}
\frac{\partial \log L}{\partial \sigma} \simeq & -\frac{1}{\sigma}\left[m+\left(1-\frac{1}{\lambda}\right) \sum_{i=i}^{m}\left(\alpha_{2 i}+\beta_{2 i} z_{i: m: n}\right) z_{i: m: n}-\sum_{i=1}^{m}\left(\gamma_{2 i}+\eta_{2 i} z_{i: m: n}\right) z_{i: m: n}\right. \\
& \left.-\sum_{i=1}^{m} r_{i}\left(\kappa_{2 i}+\delta_{2 i} z_{i: m: n}\right) z_{i: m: n}\right] \\
= & 0 .
\end{aligned}
$$

By solving the quadratic Equation (3.9) for $\sigma$, we obtain another AMLE of $\sigma$ as follows;

$$
\tilde{\sigma}_{2}=\frac{-A+\sqrt{A^{2}-4 m B}}{2 m},
$$

where

$$
\begin{aligned}
& A=\sum_{i=1}^{m} X_{i: m: n}\left[\left(1-\frac{1}{\lambda}\right) \alpha_{2 i}-\gamma_{2 i}-r_{i} \kappa_{2 i}\right], \\
& B=\sum_{i=1}^{m}\left(X_{i: m: n}\right)^{2}\left[\left(1-\frac{1}{\lambda}\right) \beta_{2 i}-\eta_{2 i}-r_{i} \delta_{2 i}\right] .
\end{aligned}
$$

The Equation (3.9) has two roots; however, one of them drops out since $\eta_{2 i}, \delta_{2 i}>0$, and $\beta_{2 i}<0$, and hence $B<0$ for $\lambda>1$. Note that the estimator $\tilde{\sigma}_{1}$ is simpler than the estimator $\tilde{\sigma}_{2}$ because $\tilde{\sigma}_{1}$ is the linear combination of available order statistics.

From the above formula, the MSEs of the proposed estimators are simulated by Monte Carlo method based on 10,000 runs for sample size $n=10(10) 40$ and various censoring schemes based on progressively Type-II censored samples. These values are given in Table 5.

\section{Estimation of the Reliability}

The probability of the survival of an item until time $t$ in the exponentiated half logistic distribution with pdf (1.1) is

$$
R(t)=1-F(t)=P[X>t]=1-\left[\frac{1-\exp \left(-\frac{t}{\sigma}\right)}{1+\exp \left(-\frac{t}{\sigma}\right)}\right]^{\lambda}, \quad t>0, \sigma, \lambda>0 .
$$

For the progressively Type-II censored data, we now propose the AMLEs and MLE of the reliability function $R(t)$ by using the proposed AMLEs $\tilde{\sigma}_{i}$ and MLE $\hat{\sigma}$ that can be used for progressively Type-II censored sample as follows;

$$
\tilde{R}_{i}(t)=1-\left[\frac{1-\exp \left(-\frac{t}{\tilde{\sigma}_{i}}\right)}{1+\exp \left(-\frac{t}{\tilde{\sigma}_{i}}\right)}\right]^{\lambda}, \quad i=1,2 \quad \text { and } \quad \hat{R}(t)=1-\left[\frac{1-\exp \left(-\frac{t}{\hat{\sigma}}\right)}{1+\exp \left(-\frac{t}{\hat{\sigma}}\right)}\right]^{\lambda} .
$$

From the Equation (4.2), the MSEs of these estimators are simulated by Monte Carlo method (based on 10,000 Monte Carlo runs) for sample size $n=30$ and $m=20$ with progressive censoring 
Table 1: Failure log times to breakdown of an insulating fluid testing experiment

\begin{tabular}{cccccccc}
\hline \hline 0.270027 & 1.02245 & 1.15057 & 1.42311 & 1.54116 & 1.57898 & 1.8718 & 1.9947 \\
\hline 2.08069 & 2.11263 & 2.48989 & 3.45789 & 3.48186 & 3.52371 & 3.60305 & 4.28895 \\
\hline \hline
\end{tabular}

Table 2: Critical values and test statistic for the Kolmogorov test

\begin{tabular}{lccc}
\hline \hline & $\hat{\sigma}$ & $\tilde{\sigma}_{1}$ & $\tilde{\sigma}_{2}$ \\
\hline Estimates & 1.52990 & 0.95721 & 0.95896 \\
Critical Value $(\alpha=0.05)$ & 0.59207 & 0.59358 & 0.59346 \\
Test statistic $D_{n}$ & 0.25975 & 0.44367 & 0.44286 \\
\hline \hline
\end{tabular}

Table 3: Progressively Type-II censored data

\begin{tabular}{ccccccccc}
\hline \hline$i$ & 1 & 2 & 3 & 4 & 5 & 6 & 7 & 8 \\
\hline$x$ & 0.270027 & 1.02245 & 1.15057 & 1.57898 & 2.11263 & 2.48989 & 3.60305 & 4.28895 \\
\hline$r_{i}$ & 0 & 0 & 2 & 3 & 0 & 3 & 0 & 0 \\
\hline \hline
\end{tabular}

Table 4: The MLEs and AMLEs of $\sigma$ and $R(t)$ when $\lambda=1$

\begin{tabular}{cccccc}
\hline \hline$\hat{\sigma}$ & $\tilde{\sigma}_{1}$ & $\tilde{\sigma}_{2}$ & $\hat{R}(t)$ & $\tilde{R}_{1}(t)$ & $\tilde{R}_{2}(t)$ \\
\hline 1.30324 & 0.95472 & 0.95668 & 0.81049 & 0.74397 & 0.74447 \\
\hline \hline
\end{tabular}

scheme $(9 * 0,10,10 * 0)$ when $\lambda=2$ and 6 . For simplicity in notation, we denote the scheme $(0,0, \ldots, n-m)$ as $((m-1) * 0, n-m)$, to give you an idea, $(10 * 0)$ and $(3 * 0,2,2,0)$ denote the progressively censoring schemes $(0,0, \ldots, 0)$ and $(0,0,0,2,2,0)$, respectively. The MSEs of the proposed estimators are presented in Figure 1 and 2.

\section{Illustrative Example and Simulation Results}

In this section, we present an example to illustrate the methods and assess the performance of estimators discussed in the previous sections.

\subsection{Real data}

Consider the data given by Nelson (1982), represents failure log times to breakdown of an insulating fluid testing experiment (see Table 1). This data has been utilized by many authors such as, Balakrishnan and Kannan (2001), Balakrishnan et al. (2004), and Alaboud (2009). We apply the Kolmogorov test to examine whether the data follow an exponentiated half logistic distribution with unknown scale parameter. We obtained the MLE and AMLEs of the scale parameter $\sigma$ when the shape parameter $\lambda=1$. Using these results, we create the critical values for the Kolmogorov test statistic for an exponentiated half logistic distribution by Monte Carlo method. Finally, we also obtain the values of the Kolmogorov test statistics $D_{n}$. These values are given in Table 2. Since the critical values at the significance level of $\alpha=0.05$ exceed the corresponding values of the test statistics, we can conclude that the data follow an exponentiated half logistic distribution. In this example, we have $n=16$ and $m=8$. The observations and censoring scheme are given in Table 3. From the Equation (2.4), (3.5), and (3.10), the MLEs $\hat{\sigma}, \hat{R}(t=0.5)$ and the AMLEs $\tilde{\sigma}_{1}, \tilde{\sigma}_{2}, \tilde{R}_{1}(t), \tilde{R}_{2}(t)$ are obtained. These values are given in Table 4.

\subsection{Simulation results}

To compare the performance of the proposed AMLEs, we simulate the MSEs of all proposed estimators through Monte Carlo simulation method. Progressively Type-II censored data for sample size 
Table 5: The relative mean squared errors for the proposed estimators of the scale parameter $\sigma$

\begin{tabular}{|c|c|c|c|c|c|}
\hline \multicolumn{6}{|c|}{$\lambda=2$} \\
\hline$n$ & $m$ & censoring scheme & $\hat{\sigma}$ & $\tilde{\sigma}_{1}$ & $\tilde{\sigma}_{2}$ \\
\hline \multirow{4}{*}{10} & 10 & $(10 * 0)$ & 0.02889 & 0.02086 & 0.01458 \\
\hline & 10 & $(3 * 0,2,2,0)$ & 0.03533 & 0.02956 & 0.02169 \\
\hline & 10 & $(4,5 * 0)$ & 0.03686 & 0.03402 & 0.02246 \\
\hline & 10 & $(5,4 * 0)$ & 0.03949 & 0.03941 & 0.02562 \\
\hline \multirow{5}{*}{20} & 20 & $(20 * 0)$ & 0.01768 & 0.00938 & 0.00715 \\
\hline & 10 & $(2 * 0,1,0,2,0,2,2 * 0,5)$ & 0.02573 & 0.01814 & 0.01583 \\
\hline & 10 & $(2 * 0,3,0,2,0,2,2 * 0,3)$ & 0.02606 & 0.01783 & 0.01501 \\
\hline & 10 & $(5,2 * 0,5,6 * 0)$ & 0.02787 & 0.01934 & 0.01390 \\
\hline & 5 & $(0,5,0,10,0)$ & 0.03690 & 0.03358 & 0.02766 \\
\hline \multirow{6}{*}{30} & 30 & $(30 * 0)$ & 0.01279 & 0.00587 & 0.00468 \\
\hline & 20 & $(9 * 0,10,10 * 0)$ & 0.01674 & 0.00889 & 0.00695 \\
\hline & 20 & $(3 * 0,5,3 * 0,5,12 * 0)$ & 0.01711 & 0.00906 & 0.00700 \\
\hline & 20 & $(2 * 0,10,17 * 0)$ & 0.01743 & 0.00922 & 0.00707 \\
\hline & 15 & $(5,6 * 0,10,7 * 0)$ & 0.02221 & 0.01322 & 0.00974 \\
\hline & 15 & $(10,6 * 0,5,7 * 0)$ & 0.02124 & 0.01247 & 0.00942 \\
\hline \multirow{6}{*}{40} & 40 & $(40 * 0)$ & 0.00960 & 0.00414 & 0.00341 \\
\hline & 20 & $(8 * 0,2 * 10,10 * 0)$ & 0.01522 & 0.00939 & 0.00872 \\
\hline & 20 & $(5,16 * 0,3 * 5)$ & 0.01626 & 0.00868 & 0.00687 \\
\hline & 20 & $(3 * 0,15,7 * 0,5,8 * 0)$ & 0.01658 & 0.00880 & 0.00691 \\
\hline & 20 & $(2 * 0,4 * 5,14 * 0)$ & 0.01701 & 0.00900 & 0.00697 \\
\hline & 10 & $(0,15,2 * 0,5,0,10,3 * 0)$ & 0.02597 & 0.01739 & 0.01379 \\
\hline \multicolumn{6}{|c|}{$\lambda=4$} \\
\hline$n$ & $m$ & censoring scheme & $\hat{\sigma}$ & $\tilde{\sigma}_{1}$ & $\tilde{\sigma}_{2}$ \\
\hline \multirow{4}{*}{10} & 10 & $(10 * 0)$ & 0.01620 & 0.00708 & 0.00551 \\
\hline & 10 & $(3 * 0,2,2,0)$ & 0.01974 & 0.00921 & 0.00752 \\
\hline & 10 & $(4,5 * 0)$ & 0.02227 & 0.01099 & 0.00824 \\
\hline & 10 & $(5,4 * 0)$ & 0.02489 & 0.01253 & 0.00931 \\
\hline \multirow{5}{*}{20} & 20 & $(20 * 0)$ & 0.00906 & 0.00327 & 0.00272 \\
\hline & 10 & $(2 * 0,1,0,2,0,2,2 * 0,5)$ & 0.01239 & 0.00559 & 0.00512 \\
\hline & 10 & $(2 * 0,3,0,2,0,2,2 * 0,3)$ & 0.01273 & 0.00557 & 0.00500 \\
\hline & 10 & $(5,2 * 0,5,6 * 0)$ & 0.01443 & 0.00617 & 0.00495 \\
\hline & 5 & $(0,5,0,10,0)$ & 0.01908 & 0.00949 & 0.00834 \\
\hline \multirow{6}{*}{30} & 30 & $(30 * 0)$ & 0.00641 & 0.00208 & 0.00178 \\
\hline & 20 & $(9 * 0,10,10 * 0)$ & 0.00793 & 0.00291 & 0.00247 \\
\hline & 20 & $(3 * 0,5,3 * 0,5,12 * 0)$ & 0.00824 & 0.00301 & 0.00254 \\
\hline & 20 & $(2 * 0,10,17 * 0)$ & 0.00859 & 0.00313 & 0.00261 \\
\hline & 15 & $(5,6 * 0,10,7 * 0)$ & 0.01149 & 0.00451 & 0.00365 \\
\hline & 15 & $(10,6 * 0,5,7 * 0)$ & 0.01035 & 0.00405 & 0.00336 \\
\hline \multirow{6}{*}{40} & 40 & $(40 * 0)$ & 0.00504 & 0.00149 & 0.00131 \\
\hline & 20 & $(8 * 0,2 * 10,10 * 0)$ & 0.00688 & 0.00293 & 0.00279 \\
\hline & 20 & $(5,16 * 0,3 * 5)$ & 0.00739 & 0.00275 & 0.00237 \\
\hline & 20 & $(3 * 0,15,7 * 0,5,8 * 0)$ & 0.00775 & 0.00285 & 0.00243 \\
\hline & 20 & $(2 * 0,4 * 5,14 * 0)$ & 0.00802 & 0.00295 & 0.00249 \\
\hline & 10 & $(0,15,2 * 0,5,0,10,3 * 0)$ & 0.01171 & 0.00509 & 0.00441 \\
\hline \multicolumn{6}{|c|}{$\lambda=6$} \\
\hline$n$ & $m$ & censoring scheme & $\hat{\sigma}$ & $\tilde{\sigma}_{1}$ & $\tilde{\sigma}_{2}$ \\
\hline \multirow{4}{*}{10} & 10 & $(10 * 0)$ & 0.01265 & 0.00405 & 0.00332 \\
\hline & 10 & $(3 * 0,2,2,0)$ & 0.01479 & 0.00515 & 0.00441 \\
\hline & 10 & $(4,5 * 0)$ & 0.01698 & 0.00617 & 0.00492 \\
\hline & 10 & $(5,4 * 0)$ & 0.01902 & 0.00700 & 0.00555 \\
\hline
\end{tabular}




\begin{tabular}{cccccc}
\hline & 20 & $(20 * 0)$ & 0.00725 & 0.00189 & 0.00163 \\
20 & 10 & $(2 * 0,1,0,2,0,2,2 * 0,5)$ & 0.00931 & 0.00312 & 0.00292 \\
& 10 & $(2 * 0,3,0,2,0,2,2 * 0,3)$ & 0.00958 & 0.00312 & 0.00287 \\
& 10 & $(5,2 * 0,5,6 * 0)$ & 0.01091 & 0.00345 & 0.00291 \\
& 5 & $(0,5,0,10,0)$ & 0.01353 & 0.00512 & 0.00464 \\
\hline \multirow{3}{*}{30} & 30 & $(30 * 0)$ & 0.00529 & 0.00121 & 0.00107 \\
& 20 & $(9 * 0,10,10 * 0)$ & 0.00624 & 0.00164 & 0.00145 \\
& 20 & $(2 * 0,5,3 * 0,5,12 * 0)$ & 0.00648 & 0.00171 & 0.00150 \\
& 20 & $(5,6 * 0,10,7 * 0)$ & 0.00677 & 0.00179 & 0.00155 \\
& 15 & $(10,6 * 0,5,7 * 0)$ & 0.00901 & 0.00258 & 0.00218 \\
& 15 & $(8 * 0,2 * 10,10 * 0)$ & 0.00799 & 0.00228 & 0.00197 \\
\hline \hline & 40 & $(5,16 * 0,3 * 5)$ & 0.00430 & 0.00087 & 0.00078 \\
& 20 & $(3 * 0,15,7 * 0,5,8 * 0)$ & 0.00544 & 0.00164 & 0.00158 \\
& 20 & $(2 * 0,4 * 5,14 * 0)$ & 0.00575 & 0.00154 & 0.00137 \\
& 20 & $(0,15,2 * 0,5,0,10,3 * 0)$ & 0.00604 & 0.00160 & 0.00142 \\
& 20 & & 0.00624 & 0.00166 & 0.00146 \\
\hline
\end{tabular}

$n=10(10) 40$ and various censoring schemes from the standard exponentiated half logistic distribution are generated by using the algorithm presented in Balakrishnan and Sandhu (1995) when the shape parameter $\lambda$ is known. Using this data, the MSEs of the proposed AMLEs are simulated by the Monte Carlo method based on 10,000 runs for sample size $n=10(10) 40$ and various choices of censoring based on progressively Type-II censored samples with $\sigma=1$. These values are given in Table 5 .

It is observed in Table 5 that $\tilde{\sigma}_{2}$ is more efficient than $\tilde{\sigma}_{1}$ and $\hat{\sigma}$ in the sense of the MSE. As expected, the MSEs of all estimators decrease as sample size $n$ increases. For fixed sample size, the MSE decreases generally as $\lambda$ increases but increases generally as the number of unobserved or missing data $n-m$ increases.

From Figure 1 and 2 , the estimators $\tilde{R}_{1}(t)$ and $\tilde{R}_{2}(t)$ are generally more efficient than $\hat{R}(t)$ for $\lambda=2$ and 6, but the MSEs of the estimators $\tilde{R}_{1}(t)$ and $\hat{R}(t)$ are almost same when $R(t)>0.9$. For $\lambda=2$ and $m=20$, the estimator $\tilde{R}_{1}(t)$ is generally more efficient than $\tilde{R}_{2}(t)$ and $\hat{R}(t)$ when $R(t)<0.4$, but the estimator $\tilde{R}_{2}(t)$ is generally more efficient than $\tilde{R}_{1}(t)$ and $\hat{R}(t)$ when $R(t) \geq 0.4$. For $\lambda=6$ and $m=20$, the estimator $\tilde{R}_{1}(t)$ is generally more efficient than $\tilde{R}_{2}(t)$ and $\hat{R}(t)$ when $R(t)<0.6$; however, the estimator $\tilde{R}_{2}(t)$ is generally more efficient than $\tilde{R}_{1}(t)$ and $\hat{R}(t)$ when $R(t) \geq 0$.6. Therefore, $\tilde{R}_{1}(t)$ is more efficient than $\tilde{R}_{2}(t)$ when $R(t)$ is small but $\tilde{R}_{2}(t)$ is more efficient than $\tilde{R}_{1}(t)$ when $R(t)$ is large.

\section{Concluding Remarks}

In this paper we propose the approximate maximum likelihood estimators(AMLEs) of the scale parameter $\sigma$ and reliability function $R(t)$ in an exponentiated half logistic distribution based on progressively Type-II censored samples when the shape parameter $\lambda$ is known. The MLEs are also obtained. Some numerical methods must be employed since the MLE $\hat{\sigma}$ cannot be solved explicitly; however, the AMLEs $\tilde{\sigma}_{1}$ and $\tilde{\sigma}_{2}$ are explicit estimators. The MLEs $\hat{\sigma}$ and $\hat{R}(t)$ are compared with AMLEs in terms of the MSE. We can see that proposed AMLEs have better performance than their corresponding MLEs for the considered cases.

When $\lambda=1$, the exponentiated half logistic distribution is the half logistic distribution that has been studied by many authors. Therefore, it will be a meaningful to study for the case of known shape parameter. Since it is difficult to obtain the AMLE of the shape parameter $\lambda$ by using the approximate maximum likelihood estimation method in this paper, this paper is restricted within known shape parameter. We will also study the estimation of the shape parameter by using Bayesian method. 


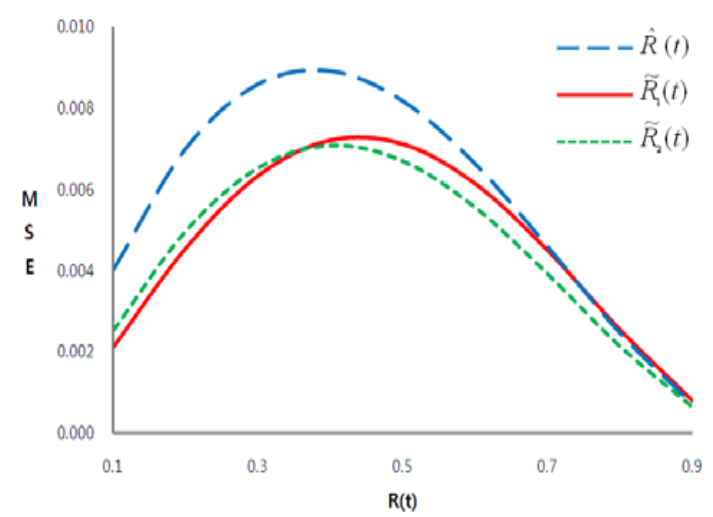

Figure 1: The relative MSEs of $\tilde{R}_{i}(t)$ and $\hat{R}(t)$ when $\lambda=2[m=20,(9 * 0,10,10 * 0)]$

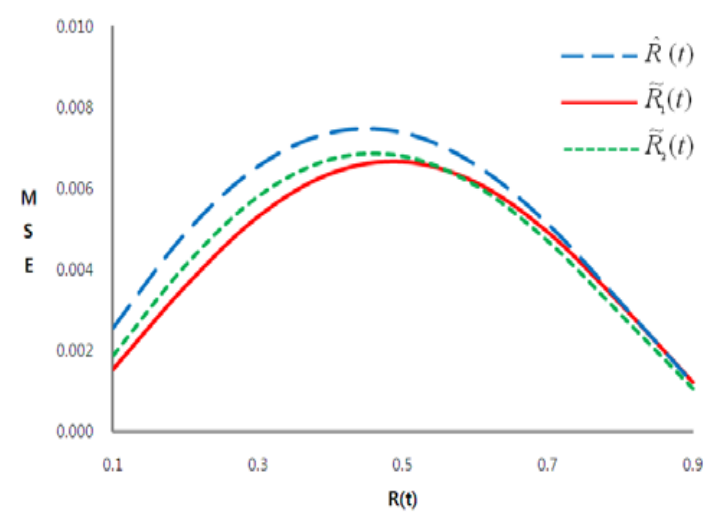

Figure 2: The relative MSEs of $\tilde{R}_{i}(t)$ and $\hat{R}(t)$ when $\lambda=6[m=20,(9 * 0,10,10 * 0)]$

\section{References}

Alaboud, F. M. (2009). Bayesian estimations for the extreme value distribution using progressive censored data and asymmetric loss, International Mathematical Forum, 8, 1603-1622.

Arora, S. H., Bhimani, G. C. and Patel, M. N. (2010). Some results on maximum likelihood estimators of parameters of generalized half logistic distribution under Type-I progressive censoring with changing, International Journal of Contemporary Mathematical Sciences, 5, 685-698.

Balakrishnan, N. (1989). Approximate MLE of the scale parameter of the Rayleigh distribution with censoring, IEEE Transactions on Reliability, 38, 355-357.

Balakrishnan, N. and Kannan, N. (2001). Point and interval estimation for the logistic distribution based on progressively Type-II censored samples, In Handbook of Statistics, Balakrishnan, N. and Rao, C. R., Eds., 20, 431-456.

Balakrishnan, N., Kannan, N., Lin, C. T. and Ng, H. K. T. (2003). Point and interval estimation for Gaussian distribution based on progressively Type-II censored samples, IEEE Transactions on Reliability, 52, 90-95.

Balakrishnan, N., Kannan, N., Lin, C. T. and Wu, S. J. S. (2004). Inference for the extreme value distribution under progressively Type-II censoring, Journal of Statistical Computation and Simulation, 74, 25-45. 
Balakrishnan, N. and Puthenpura, N. (1986). Best linear unbiased estimators of location and scale parameters of the half logistic distribution, Journal of Statistics and Computer Simulation, 25, 193-204.

Balakrishnan, N. and Sandhu, R. A. (1995). A simple simulational algorithm for generating progressively Type-II censored samples, The American Statistician, 49, 229-230.

Balakrishnan, N. and Wong, K. H. T. (1991). Approximate MLEs for the location and scale parameters of the half-logistic distribution with Type-II right censoring, IEEE Transactions on Reliability, 40, 140-145.

Han, J. T. and Kang, S. B. (2008). Estimation for the double Rayleigh distribution based on multiply Type-II censored samples, Communications of the Korean Statistical Society, 15, 367-378.

Kang, S. B., Cho, Y. S. and Han, J. T. (2008). Estimation for the half logistic distribution under progressively Type-II censoring, Communications of the Korean Statistical Society, 15, 815-823.

Kang, S. B., Cho, Y. S. and Han, J. T. (2009). Estimation for the half logistic distribution based on double hybrid censored samples, Communications of the Korean Statistical Society, 16, 10551066.

Lee, H. J., Han, J. T. and Kang, S. B. (2008). Estimation for a triangular distribution based on multiply Type-II censored samples, Journal of Korean Data \& Information Science Society, 19, 319-330.

Lin, C. T., Wu, S. J. S. and Balakkrishnan, N. (2006). Inference for log- gamma distribution based on progressively Type-II censored data, Communication in Statistics-Theory and Methods, 35, 1271-1292.

Nelson, W. B. (1982). Applied Life Data Analysis, John Willey \& Sons, New York.

Seo, E. H. and Kang, S. B. (2007). AMLEs for Rayleigh distribution based on progressively Type-II censored data, The Korean Communications in Statistics, 14, 329-344. 